\title{
Imaging of the Dust Acoustic Wave to Explore Synchronization
}

\author{
Wellalage Don Suranga Ruhunusiri and John Goree
}

\begin{abstract}
A self-excited dust acoustic wave is synchronized by sinusoidal modulation of the ion density, and the wave is imaged by planar laser-light scattering. Space-time diagrams based on the images reveal how the nonlinearity of the plasma's response causes the wave's frequency to be synchronized to a multiple of $0.5,1,2$, or 3 of the modulation frequency. Space-time diagrams also reveal wave front merging as is observed for a wide range of modulation frequencies.
\end{abstract}

Index Terms-Dusty plasmas, plasma waves.

$\mathbf{T}$ HE dust acoustic wave (DAW) is a plasma wave that an experimenter can observe with the naked eye. This wave is analogous to an ion acoustic wave and propagates in a dusty plasma containing highly charged particles of solid matter (dust) in addition to electrons, ions, and neutral gas atoms. Compressions and rarefactions of the dust particles have such a high amplitude, and they propagate so slowly, that they are easy to view and image by laser light scattering. Due to the low charge-to-mass ratio of the dust particles, the wave frequency is typically only a few tens of hertz. The wave is self-excited by an ion-streaming instability [1].

We performed an experiment to observe nonlinear phenomena, which occur because the wave grows to a large amplitude. We observed wave synchronization where the wave's frequency changes in response to an external modulation of the plasma conditions [2]-[4]. We study the same synchronization phenomenon as in [4], but here we rely on space-time diagrams, prepared as in [5], as our analysis method.

An argon glow discharge plasma was ignited by applying a 13.56-MHz radio frequency voltage with $57 \mathrm{~V}$ peak-to-peak amplitude to a horizontal lower electrode [Fig. 1(a)]. Dust particles, which were $4.8-\mu \mathrm{m}$ melamine formaldehyde spheres, were dropped into the plasma using a dispenser with a single hole. The dust particles were negatively charged by collecting more electrons than ions, and they levitated under the action of naturally occurring electric fields. To vertically elongate the dust cloud, we used a glass box that enhanced the horizontal component of the electric field. The vertical component of this electric field drove a downward ion flow, which was the energy source for exciting the DAW. The 120-mTorr gas pressure was low enough that gas damping did not

Manuscript received November 19, 2013; accepted April 28, 2014. Date of publication May 16, 2014; date of current version October 21, 2014. This work was supported in part by the National Aeronautics and Space Administration and in part by the National Science Foundation.

The authors are with the Department of Physics and Astronomy, University of Iowa, Iowa City, IA 52242 USA (e-mail: suranga-ruhunusiri@uiowa.edu; john-goree@uiowa.edu).

Digital Object Identifier 10.1109/TPS.2014.2321105 prevent the DAW from growing to large amplitudes with significant nonlinearities. The natural frequency of the wave, meaning its frequency without any modulation present, was $20.5 \mathrm{~Hz}$.

Our primary diagnostic was planar laser-light scattering for imaging the dust cloud [6]. The intensity of the scattered light was proportional to the dust number density $n_{\mathrm{d}}$, because the dust cloud was optically thin. A vertical cross section of the cloud was illuminated with a sheet of 532-nm laser light. A movie was recorded at 256 frames/s using a Phantom v5.2 camera. A still image is shown in Fig. 1(b). We analyzed the intensity within a region of interest (ROI) [Fig. 1(b)]. The wavefronts were planar in the ROI, so that we can average the intensity over the ignorable coordinate $x$. This analysis yielded a time-resolved and height-resolved record of the scattering intensity $I(z, t)$, which is proportional to the dust number density $n_{\mathrm{d}}$. We then calculated the fractional fluctuation of $n_{\mathrm{d}}$ as $\tilde{N}(z, t)=\left[n_{\mathrm{d}}(z, t)-\left\langle n_{\mathrm{d}}(z)\right\rangle\right] /\left\langle n_{\mathrm{d}}(z)\right\rangle$, where the angle brackets denote a time average.

We applied a modulation to synchronize the wave. A $46 \mathrm{~V}$ peak-to-peak sinusoidal waveform was applied to a grid, which also had a bias of $-60 \mathrm{~V}$ so that it collected ions. We verified that the resulting ion density modulation was sinusoidal using a Langmuir probe. Further details of the setup are reported in [4]. We investigated the nonlinear response of the wave to different modulation frequencies $f_{\mathrm{dr}}$ in steps of $1 \mathrm{~Hz}$ from 2 to $45 \mathrm{~Hz}$, which was the highest frequency at which we could attain synchronization.

We present the results for the dust number density fluctuations $\tilde{N}(z, t)$ as space-time diagrams [Fig. 1(c)-(g)]. The wavefronts are seen as sloping bands that are dark for compression and light for rarefaction. The period of the observed wave is determined by the temporal spacing between wave crests, yielding the observed frequency of the wave $f_{\mathrm{w}}$.

One effect of nonlinearity is seen by noting that the wave frequency $f_{\mathrm{w}}$ is generally not the same as the natural frequency $20.5 \mathrm{~Hz}$. The sinusoidal modulation has caused the wave's frequency to change. For example, in Fig. 1(c), applying an $f_{\mathrm{dr}}=8-\mathrm{Hz}$ modulation caused the wave's frequency to change to $f_{\mathrm{w}}=24 \mathrm{~Hz}$. The frequency usually changes by a rational fraction, which in this case was 3:1.

Another effect of nonlinearity is wavefront merging, as was reported in [2]. This is observed in Fig. $1(\mathrm{~g})$ at $z=7 \mathrm{~mm}$, where two downward-propagating wavefronts merge into one, causing the wavelength and wave period to double.

Finally, another effect of nonlinearity, which we detected in [4] using a frequency spectrum, can also be seen in the 

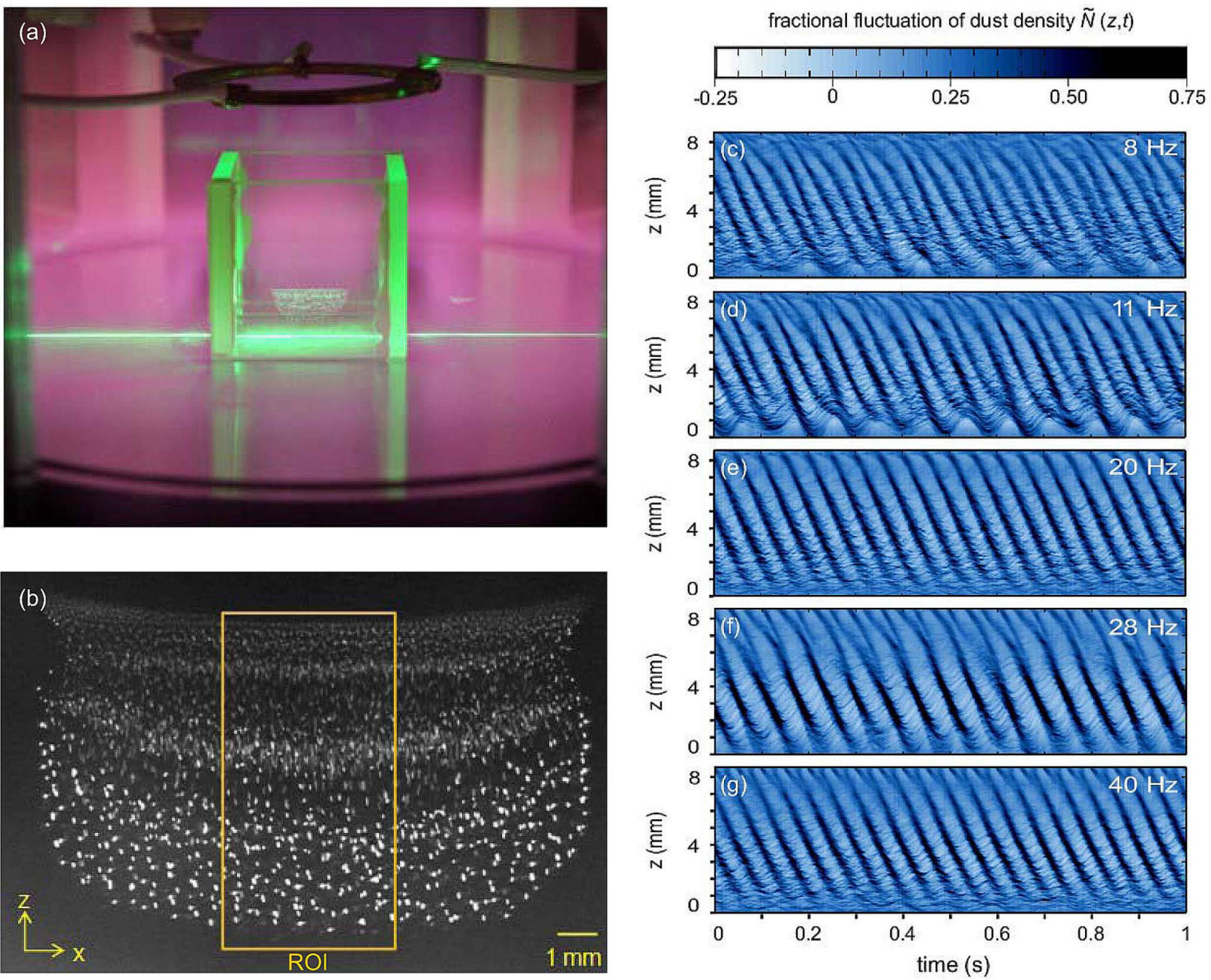

Fig. 1. (a) Experimental setup. An argon plasma is sustained by radio-frequency voltage applied to the large horizontal electrode. A vertical dc electric field drives a downward ion flow and provides an upward levitation force for $4.8-\mu \mathrm{m}$ dust particles. A 3-cm glass cube, open on top and bottom, modifies dc electric fields to enhance horizontal confinement of the dust. The dust cloud can be seen as a hemispherical volume above the bottom of the cube. A ring-shaped electrode above the glass box is used to modulate the plasma density sinusoidally at a desired frequency $f_{\mathrm{dr}}$. A vertical cross section of the dust cloud is illuminated by a sheet of 532-nm laser light. (b) High-resolution high-speed image of the dust cloud showing compressions and rarefactions of the downward propagating DAW. (c)-(g) Space-time diagrams of the wave for various $f_{\mathrm{dr}}$, showing the fractional fluctuation of dust density $N(z, t)$, as determined by laser light scattering in the ROI shown in (b).

space-time diagram of Fig. 1(f) at $z=6 \mathrm{~mm}$. Here, the downward propagating waves sometimes merge, but sometimes they do not, so that the frequency changes by a nonrational fraction. In [4], we referred to this phenomenon as a nonharmonic state.

\section{REFERENCES}

[1] M. Rosenberg, "Ion- and dust-acoustic instabilities in dusty plasmas," Planetary Space Sci., vol. 41, no. 3, pp. 229-233, 1993.

[2] I. Pilch, T. Reichstein, and A. Piel, "Synchronization of dust density waves in anodic plasmas," Phys. Plasmas, vol. 16, no. 12, p. 123709 , 2009.
[3] T. Trottenberg, D. Block, and A. Piel, "Dust confinement and dust-acoustic waves in weakly magnetized anodic plasmas," Phys. Plasmas, vol. 13, no. 4, p. 042105, 2006.

[4] W. D. S. Ruhunusiri and J. Goree, "Synchronization mechanism and Arnold tongues for dust density waves," Phys. Rev. E, vol. 85, no. 4, p. 046401, 2012.

[5] T. M. Flanagan and J. Goree, "Observation of the spatial growth of self-excited dust-density waves," Phys. Plasmas, vol. 17, no. 12, p. 123702, 2010.

[6] J. D. Williams, E. Thomas, Jr., and L. Marcus, "Observations of vertically propagating driven dust acoustic waves: Finite temperature effects," Phys. Plasmas, vol. 15, no. 4, p. 043704, 2008. 